\title{
Endorectal Advancement Flap With Muscular Plication in Anovaginal and Anterior Perineal Fistulas
}

\author{
Axel Egal, Isabelle Etienney, Patrick Atienza \\ ${ }^{1}$ Department of Proctology, Groupe Hospitalier Diaconesses Croix Saint-Simon, Paris, France
}

Purpose: Endorectal mucosal advancement flap with muscular plication can ensure complete closure of anovaginal fistulas and preserve continence. The aim of this retrospective study was to show indications might be broadened to include anoperineal fistulas.

Methods: This retrospective study gathered all available data from patients with anovaginal or anterior perineal fistulas who underwent transanal advancement flap repair with muscular plication. A loose seton was passed in the fistula track prior to surgery in all patients. Fistula healing was defined as fistula closure during proctological examination associated with complete resolution of symptoms.

Results: Thirty-five patients were included from January 2011 to March 2017. Causes of fistula were various, mostly postoperative $(34.3 \%, \mathrm{n}=12)$, obstetrical $(17.1 \%, \mathrm{n}=6)$ and inflammatory $(14.3 \%, \mathrm{n}=5)$. Success rate was $65.2 \%$. Fistula healing was obtained in $60.0 \%$ of patients with Crohn disease in remission. Closure rate was higher in anterior perineal fistulas (89.0\%) than in anovaginal fistulas (63.6\%) even if it did not reach statistical significance. Slight fecal continence disorders were noted in 2 women (5.7\%).

Conclusion: This study demonstrates the efficacy of transanal advancement flap repair with muscular plication for anovaginal and anterior perineal fistulas. Similar closure rates and smaller postoperative incontinence rates compared to the classical technique make this surgery an optimal solution whose efficacy appears to be sustainable over time.

Keywords: Rectovaginal fistula; Anovaginal fistula; Endorectal advancement flap; Muscular plication; Anal continence

\section{INTRODUCTION}

Anovaginal fistula (AVF) is defined by an abnormal connection, frequently epithelialized, between the anorectum and the vagina. It must not be confused with the anterior perineal fistula (APF) defined as an anal fistula with an external opening located be-

Received: Nov 19, 2019 - Revised: Apr 8, 2020 - Accepted: Apr 10, 2020 Correspondence to: Axel Egal, M.D.

Department of Proctology, Groupe Hospitalier Diaconesses Croix Saint-Simon, 125 rue d'Avron, Paris 75020, France

Tel: +33-1-44-64-20-64, Fax: +33-1-44-64-33-17

E-mail:AEgal@hopital-dcss.org

ORCID: https://orcid.org/0000-0002-7265-5781

\section{- This manuscript has been a poster meeting presentation (Journées Francophones d'Hépatologie-Gastroentérologie et d'Oncologie Digestive, 22-25 March 2018, Paris, France).}

(C) 2021 The Korean Society of Coloproctology

This is an open-access article distributed under the terms of the Creative Commons Attribution NonCommercial License (https://creativecommons.org/licenses/by-nc/4.0) which permits unrestricted noncommercial use, distribution, and reproduction in any medium, provided the original work is properly cited. tween 11 and 1 hour (anal-clock system). APF and AVF share the same etiologies and must be treated accordingly [1].

Treatment is difficult, nonconsensual, and often yields poorquality results. Most of the time, surgery is required due to lack of spontaneous healing [1] and to significant impairment in quality of life. "Classical" endorectal flap is an available option but requires a section of the internal anal sphincter and thus can lead to impairment of anal continence [2].

In 2011, our department described a new "sphincter-sparing" technique using an exclusively mucosal and submucosal flap not requiring section of the internal anal sphincter combined with plication of the rectal muscular layer. This modified endorectal advancement flap procedure was found easy to perform and cured 15 of the 23 patients $(65.2 \%)$ in our early series without any obvious deterioration of anal continence [2].

The aim of this retrospective study of the procedure using new technical amendments was to evaluate the results in patients with AVF or APF, assessing outcome in terms of fistula closure and continence after a longer follow-up. 


\section{METHODS}

All patients treated by transanal advancement flap repair with muscular plication for AVF or APF between January 2011 and September 2017 in the department of proctology of the Diaconesses Croix Saint-Simon Hospital (Paris, France) were included in the study. Fistula was characterized as postoperative (or iatrogenic) if they occurred less than one year after a proctologic surgery (except if performed in order to treat a previous APF or AVF).

Examination under general or spinal anesthesia was systematic throughout the first operative time. All abscesses were incised and the internal anal opening was cauterized. The fistula track was catheterized and then a loose seton was left in place for at least 6 weeks until inflammation had resolved. In case of Crohn disease, medical treatment was optimized after seton insertion in order to treat the flare before advancement flap repair [3].

No colostomy was performed [4]. A residue-free diet was started 1 week before the operation and patients were given a polyethylene glycol-based mechanical bowel preparation the day before. A sorbitol enema was administrated on the morning of surgery. Endorectal advancement flap repair with muscular plication was then performed as previously described [2]. As a reminder, an anterior transverse incision was made distal to the internal opening extending to the submucosa, and a U-shaped flap consisting of mucosa and submucosa was prepared. The dissection was carried out in a cephalad direction until the entire flap could be easily advanced distally. The distal part of the flap, including the internal opening, was excised. The internal opening was closed with a fig-

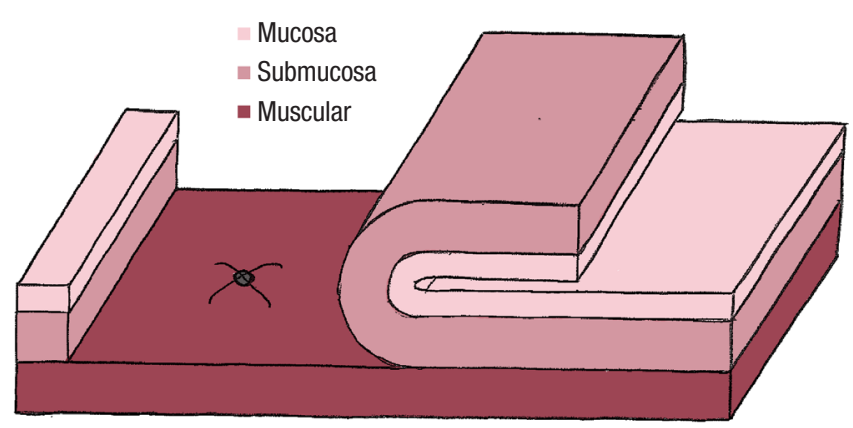

Fig. 1. Figure-of-8 stitch of the primary orifice. ure-of- 8 stitch (Fig. 1) and then a transverse plication of the muscular layer and the internal anal sphincter and/or rectal muscular layers depending on the height of the fistula, was made using an overthread suture or interrupted sutures (Fig. 2). Finally, the mucosal-submucosal flap was advanced to cover the muscular plication and closed without tension by 3 sutures distally and 1 suture laterally (Fig. 3).

To ensure its efficiency, the surgical technique underwent certain modifications and refinements over time (use of thinner sutures and switch from an overthread suture to several interrupted sutures for flap fixation, increase in flap width, and reduced duration of parenteral nutrition with cessation of port-a-cath use). These modifications are summarized in Table 1.

Postoperatively, total parenteral nutrition associated with fasting was administered for 2 to 7 days after surgery followed by a resi-

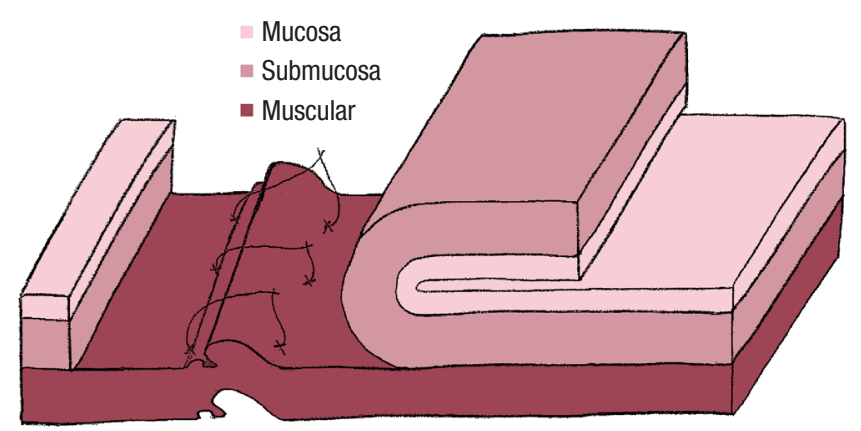

Fig. 2. Transverse plication of the muscular layer using an overthread suture.

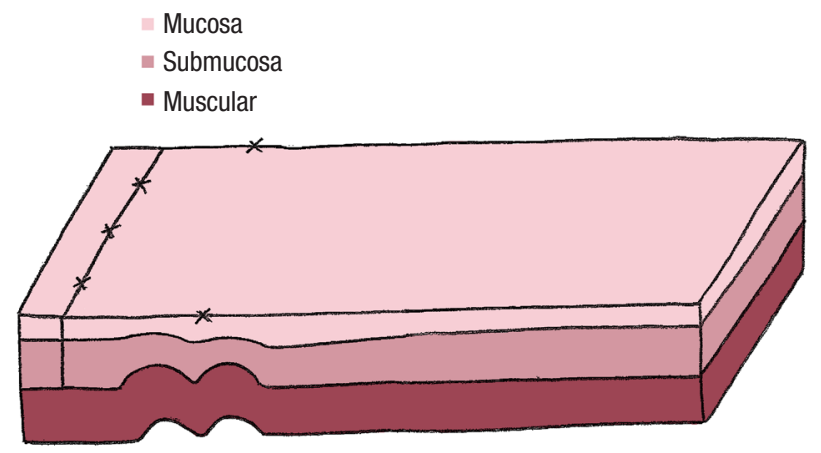

Fig. 3. Final result.

Table 1. Modifications of transanal advancement flap repair with muscular plication over time

\begin{tabular}{llllcc}
\hline Technique No. & "X" suture & Plication & Fixation & Width (cm) & PN (day) \\
\hline 1 (2011-2013) & Vicryl 3-0 & Vicryl 1 & OT, Vicryl 2-0 & 2 & Yes (7, PAC) \\
$2($ 2014-2016) & Vicryl 3-0 & Vicryl 1 & IS, Vicryl 3-0 & 2 & Yes (2) \\
$3(2017-)$ & Vicryl 3-0 & Vicryl 1 & IS, Vicryl 3-0 & 2 & Yes (2) \\
\hline
\end{tabular}

Fixation and width are related to the advancement flap.

OT, overthread suture; IS, interrupted sutures; PN, parenteral nutrition; PAC, port-a-cath.

Vicryl; Ethicon Inc., Cincinnati, OH, USA. 
due-free diet for 1 week in order to reduce the volume of stools as well as its frequency. Indeed, some studies suggest that defecation during early postoperative period may be one of the prime reasons for surgical site infections and wound dehiscence [5]. Patients were discharged on day 2. Oral antibiotic therapy (metronidazole, $1.5 \mathrm{~g}$ daily) was administered for 1 week after surgery and local antibiotics (metronidazole) were applied anally once a day for at least 1 month.

There was no specific postoperative care other than careful perineal hygiene. Patients were seen in consultation approximatively every 15 days until the third month. The first postoperative rectal examination could be performed after 9 days and the first anoscopy after 15 days. Patients were informed that 2 months after surgery vaginal intercourse would not affect healing.

Fistula healing was defined as fistula closure at the proctological follow-up examination together with complete resolution of the passage of flatus or stool per vaginam (in case of AVF). Magnetic resonance imaging was not used to assess anoperineal fistula healing because we lack a clear validated definition of the expected result: it displays low interrater reliability, especially for scoring and, moreover, persistent suspicious T1-weighted images may be present long after clinical healing [6]. Outcome was considered to be treatment failure for patients lost to follow-up. Clinical data obtained retrospectively from medical records included age at diagnosis, sex, weight and height, smoking status, Crohn disease, diabetes, history of surgery for AVF or APF, fistula localization, etiology, symptoms, complementary exams before surgery, date of surgery, pre- and postoperative continence, postoperative pain, fistula healing, type and efficacy of surgery in the event of relapse, and date of the last clinic visit recorded in the database.

Duration of postoperative follow-up was defined as time from date of surgery (advancement flap) to the date of the last followup visit. The Kaplan-Meier method was used to determine the 3-month failure rate and the log-rank test for between-group univariate comparisons. Data analysis was performed with GraphPad Prism (ver. 6, GraphPad Software, San Diego, CA, USA). Statistical significance was set at $\alpha=0.05$.

\section{RESULTS}

From January 2011 to March 2017, 34 women and 1 man were included. All fistulas were located in the lower third of the anorectum. There were 8 cases of APF (22.9\%) and 27 cases of AVF (77.1\%). Causes of fistulas were postoperative $(34.3 \%, n=12)$, obstetrical $(17.1 \%, \mathrm{n}=6)$, inflammatory $(14.3 \%, \mathrm{n}=5)$ and others (35.3\%). It should be noted that all postoperative fistulas were secondary to previous proctological surgery (for anal fistula and/ or fissure, hemorrhoids) and that there was no history of coloanal or ileoanal anastomosis.

Median time from symptom onset to diagnosis was 15 months (range, 0 to 193 months). Ten patients (28.6\%) had already undergone potentially curative fistula surgery ( 1 to 7 prior opera- tions). Median time from loose seton insertion to muscular plication was 3 months (range, 2 to 15 months). That time was extended to 11.4 months in patients with Crohn disease. The median postoperative follow-up was 31 months (range, 4 to 72 months).

Success rate was $68.6 \%(24 / 35)$. Closure rate was higher in patients with APF (89.0\%) than AVF (63.6\%) $(\mathrm{P}=0.760)$. Among the 11 patients with surgical failure, fistula healing was achieved secondarily in 5 using a new muscular plication (same method, $\mathrm{n}=3)$, perineoproctectomy $(\mathrm{n}=1)$ and a cutting seton $(\mathrm{n}=1)$, bringing the global success rate to $82.9 \%$ (29 of 35). The 6 other patients were lost to follow-up and thus considered as treatment

Table 2. Risk factors for 3-month postoperative failure in transanal advancement flap repair with muscular plication

\begin{tabular}{|c|c|c|c|}
\hline Variable & Total & 3-Mo failure & P-value \\
\hline \multicolumn{4}{|l|}{ Age (yr) } \\
\hline$<40$ & $23(65.7)$ & 34.8 & 0.770 \\
\hline$>40$ & 12 (34.3) & 33.3 & \\
\hline \multicolumn{4}{|l|}{ Active smoking } \\
\hline Yes & $16(45.7)$ & 35.7 & 0.840 \\
\hline No & 19 (54.3) & 27.7 & \\
\hline \multicolumn{4}{|l|}{ Overweight ${ }^{\mathrm{a}}$} \\
\hline Yes & 22 (62.9) & 36.4 & 0.360 \\
\hline No & 13 (37.1) & 23.1 & \\
\hline \multicolumn{4}{|l|}{ Previous surgery } \\
\hline Yes & 10 (28.6) & 33.3 & 0.230 \\
\hline No & 25 (71.4) & 36.0 & \\
\hline \multicolumn{4}{|l|}{ Crohn disease } \\
\hline Yes & $5(14.3)$ & 40.0 & NA \\
\hline No & 30 (85.7) & 30.0 & \\
\hline \multicolumn{4}{|l|}{ Ultrasound ASD } \\
\hline Yes & $9(25.7)$ & 45.0 & 0.400 \\
\hline No & $26(74.3)$ & 19.0 & \\
\hline \multicolumn{4}{|l|}{ Fistula type } \\
\hline AVF & 27 (77.1) & 36.0 & 0.760 \\
\hline APF & 8 (22.9) & 12.5 & \\
\hline \multicolumn{4}{|l|}{ Type of anesthesia } \\
\hline General & 29 (82.9) & 34.5 & 0.410 \\
\hline Spinal & $6(17.1)$ & 16.7 & \\
\hline \multicolumn{4}{|l|}{ Surgical technique } \\
\hline Technique No. 1 & $6(17.1)$ & 33.3 & \\
\hline Technique No. 2 & 18 (51.4) & 27.8 & NA \\
\hline Technique №. 3 & 11 (31.4) & 27.2 & \\
\hline
\end{tabular}

Values are presented as number (\%) or percent only.

ASD, anal sphincter defect; NA, non-assessed.

aBody mass index $>25 \mathrm{~kg} / \mathrm{m}^{2}$. 


\section{Coloproctology Axel Egal, et al.}

failure.

Healing rate was unrelated to history of previous surgery, age, overweight, active smoking, or Crohn disease (Table 2). In patients with Crohn disease, less than half (40.0\%) had an associated clinical luminal flare (rectal, colonic and/or ileal). Fistula healing was obtained in $60.0 \%$ of patients with Crohn disease (3 of 5) for whom surgery was combined with a change in medical treatment (switch to anti-tumor necrosis factor in $80 \%$ ) versus $70.0 \%$ of patients with noninflammatory fistula (21 of 30). The difference was not significant.

A partial defect of the internal and/or external anal sphincter was disclosed on the preoperative ultrasound in 9 patients (25.7\%). In these patients, closure rate was lower $(55.0 \%)(\mathrm{P}=0.400)$, especially when the external anal sphincter was involved (closure rate, $25.0 \%)$. In the 6 patients operated under spinal anesthesia (2 APF and $4 \mathrm{AVF}$ ), healing rate appeared to be higher (82.9\%) (Table 2).

Early postoperative pain (within 15 days after surgery) was noted in 17\% of patients (none of the patients received class 3 analgesics). Fistula-related symptoms still present 1 month after surgery were not synonymous with technical failure. However, persistence of symptoms 3 months after surgery was associated with long-term failure of fistula closure. Slight fecal continence disorders (soiling and flatus incontinence only) were noted in 2 women (5.7\%). These patients were naïve of any anal surgery before appearance of the fistula.

\section{DISCUSSION}

This series confirms the efficacy of the endorectal mucosal advanced flap with muscular plication for the treatment of AVF and shows that this technique can be applied to APF as a sphinctersparing method preserving the general architecture of the anal canal. The global healing rate (68.6\%) was similar to those reported in the literature for "classical" endorectal flaps. Similarly, the lower closing rates we observed in inflammatory fistulas and better results in APF than in AVF have also been reported by others [7-9]. The only publication we found examining results both in AVF and in APF, and both in inflammatory disease and no fistula cases, is that of Sonoda et al. [8] who had a global healing rate of $63.6 \%$ with the same risk factors of failure as observed here.

Technical amendments made over time (thinner sutures, shorter duration of parenteral nutrition, not using port-a-cath; Table 1) apparently did not alter the results compared with our earlier series [2]. In the new series with more patients and a longer followup, there was a greater proportion of fistulas caused by infection (cryptoglandular) or iatrogenic events, yet the healing rates were comparable. All patients had a loose seton to drain the fistula tract before surgery, a technique known to improve results $[10,11]$.

No colostomy was performed because even if such a gesture could have improved our results, it would have dramatically worsened quality of life and increased morbidity.

We did not have postoperative infection in that series. In our opinion, it was due to various factors: previous drainage of the fistula with a loose seton, residue-free diet and bowel preparation in order to reduce the number of stools after surgery, use of thinner sutures, use of interrupted sutures in order to close the flap, use of postoperative antibiotherapy and especially a very careful perineal hygiene after the surgery.

We were unable to demonstrate that overweight (body mass index, $>25 \mathrm{~kg} / \mathrm{m}^{2}$ ) or active smoking affect results as observed by others $[9,12]$, perhaps due to our small sample size. Results did however appear to be better with spinal anesthesia. The fact that general anesthesia can potentially jeopardize results because of insufficient relaxation and bleeding-hampered dissection was noted as early as 1948 by Laird [13]. Healing rates were also lower among patients with a preoperative ultrasound showing an anal sphincter defect. This was already noticed by Tsang et al. [14] in 1998 who found less satisfactory results in patients with anal sphincter defects, probably due to tissue fragility making flap achievement more complex. Again, probably due to our small sample size, these 2 effects were not significant in our series.

Our median follow-up (31 months) is one of the longest reported in the literature in patients with an advancement flap, suggesting that this modified technique provides sustainable results over time. Moreover, we also noticed that fistula-related symptoms lasting up to one month after surgery were not synonymous with technical failure. Within one month, the covering flap is possibly not well-cicatrized and thus perfect closure of fistula is not effective at this point. Besides, resorbable stitches can still be present and generate a mucous rectal discharge. This observation should encourage clinicians to wait a few months (at least 3 or 4 months) before incorrectly diagnose a surgical failure and consider a new surgery.

Specific techniques may vary between institutions and surgeons, but one of the final objectives of surgery for AVF and APF remains the maintenance of fecal continence. Nevertheless, the changes produced as a consequence of creating the flap, i.e., loss of rectal sensitivity and probably prolonged use of an anal retractor, could adversely alter anal continence. While the best results for full- and partial-thickness flaps are described in terms of recurrence, it seems that continence disorders increase with flap thickness. In the recent meta-analysis of Balciscueta et al. [15], the pooled rate of fecal incontinence (11 studies, 622 patients) was $13.3 \%$ (95\% confidence interval [CI], 8 to 18.6 ) and ranged from $0 \%$ to $51 \%$. The pooled rate of anal incontinence in mucosal flaps was $10.3 \%$ (95\% CI, 2.7 to 18) in partial-thickness flaps $14.1 \%$ ( $95 \%$ CI, 5.3 to 23) and in full-thickness flaps 20.4\% (95\% CI, 14.2 to 26.6) [15].

In the previous study of our department, the Wexner incontinence score was assessed in 15 patients and improved from 6 (range, 3 to 13) to 1 (range, 0 to 6) after surgery. In the present series, slight fecal continence disorders (soiling and flatus incontinence only) appeared to be infrequent with only 2 patients out of $35(5.7 \%)$ reporting these symptoms. This leads to the conclusion 
Table 3. Comparisons and combinations with our previous study

\begin{tabular}{lccc}
\hline Characteristic & Previous study $^{\mathrm{a}}$ & Present study & Combination \\
\hline No. of patients & 23 & 35 & 58 \\
Age (yr) & $45.5(28-78)$ & $39(24-67)$ & $41.5(24-78)$ \\
Etiology & & & \\
$\quad$ Obstetrical & $5(21.7)$ & $6(17.1)$ & $11(19.0)$ \\
$\quad$ Inflammatory & $7(30.4)$ & $5(14.3)$ & $12(20.7)$ \\
$\quad$ CG/iatrogenic & $11(47.8)$ & $12(34.3)$ & $23(39.7)$ \\
Active smoking & $3(13.0 \%)$ & $16(45.7)$ & $19(32.8)$ \\
Previous surgery & $13(56.5 \%)$ & $10(28.6)$ & $23(39.7)$ \\
Seton duration (mo) & $5.7(1.4-31)$ & $3(1-15)$ & $4(1-31)$ \\
Surgery & & & \\
$\quad$ Healing rate & $15(65.2)$ & $24(68.6)$ & $39(67.2)$ \\
Follow-up (mo) & $14(2-67)$ & $31(4-72)$ & $24(2-72)$ \\
\hline
\end{tabular}

Values are presented as number only, median (range), or number (\%).

CG, cryptoglandular.

${ }^{a}$ De Parades et al. [2].

that our modified technique provides similar closure rates and fewer, less bothersome postoperative continence disorders than full- and partial-thickness flaps.

This observation must, however, be considered with caution given the presence of some major drawbacks. Firstly, the small number of patients and the retrospective nature of our series are major bias. Consequently, patients are very heterogeneous: they differ by type of fistulous tract, diameter of the thread used, suture methods, and duration of postoperative hospitalization. Moreover, continence was not systematically assessed with any validated scale and was based only on patients complains. This may result in significant underestimation. In our opinion, the relatively high rate of patients who were lost to follow-up $(17.1 \%, n=6)$ is not of concern because it was considered as treatment failure and thus could not have led to an overestimation of the fistula healing rate.

Dates from the present study were compared and summarized together with our department's previous series [2] in Table 3.

This study confirms the simplicity and the efficacy of transanal advancement flap repair with muscular plication for AVF and shows that this technique can be used for APF. Fistula closure rates were similar to those obtained with the classical technique requiring section of the smooth anal sphincter and postoperative incontinence rates were lower, suggesting this surgery can be an optimal solution providing sustained efficacy over time.

\section{CONFLICT OF INTEREST}

No potential conflict of interest relevant to this article was reported.

\section{REFERENCES}

1. Das B, Snyder M. Rectovaginal fistulae. Clin Colon Rectal Surg 2016;29:50-6.

2. De Parades V, Dahmani Z, Blanchard P, Zeitoun JD, Sultan S, Atienza P. Endorectal advancement flap with muscular plication: a modified technique for rectovaginal fistula repair. Colorectal Dis 2011;13:921-5.

3. DeLeon MF, Hull TL. Treatment strategies in Crohn's-associated rectovaginal fistula. Clin Colon Rectal Surg 2019;32:261-7.

4. Pinto RA, Peterson TV, Shawki S, Davila GW, Wexner SD. Are there predictors of outcome following rectovaginal fistula repair? Dis Colon Rectum 2010;53:1240-7.

5. Gao S, Zheng Y, Liu X, Tian Z, Zhao Y. Effect of early fasting and total parenteral nutrition support on the healing of incision and nutritional status in patients after sacrectomy. Orthop Traumatol Surg Res 2018;104:539-44.

6. Brochard C, Landemaine A, L'Heritier AM, Dewitte MP, Tchoundjeu B, Rohou T, et al. Anal fistulas in severe perineal Crohn's disease: MRI assessment in the determination of long-term healing rates. Inflamm Bowel Dis 2018;24:1612-8.

7. Ellis CN, Clark S. Effect of tobacco smoking on advancement flap repair of complex anal fistulas. Dis Colon Rectum 2007;50:45963.

8. Sonoda T, Hull T, Piedmonte MR, Fazio VW. Outcomes of primary repair of anorectal and rectovaginal fistulas using the endorectal advancement flap. Dis Colon Rectum 2002;45:1622-8.

9. Lowry AC, Thorson AG, Rothenberger DA, Goldberg SM. Repair of simple rectovaginal fistulas: influence of previous repairs. Dis Colon Rectum 1988;31:676-8.

10. Milito G, Lisi G, Venditti D, Campanelli M, Aronadio E, Grande M. Surgical treatment of rectovaginal fistula in Crohn's disease: a tertiary center experience. Surg Technol Int 2017;30:113-6.

11. Manne A, Ahmed MB, Malik TA. Predictors of outcome of rectovaginal fistula surgery in women with Crohn's disease. J Clin Med Res 2016;8:126-9.

12. Van Onkelen RS, Gosselink MP, Thijsse S, Schouten WR. Predictors of outcome after transanal advancement flap repair for high transsphincteric fistulas. Dis Colon Rectum 2014;57:1007-11.

13. Laird DR. Procedures used in treatment of complicated fistulas. Am J Surg 1948;76:701-8.

14. Tsang CB, Madoff RD, Wong WD, Rothenberger DA, Finne CO, Singer D, et al. Anal sphincter integrity and function influences outcome in rectovaginal fistula repair. Dis Colon Rectum 1998; 41:1141-6.

15. Balciscueta Z, Uribe N, Balciscueta I, Andreu-Ballester JC, Garcia-Granero E. Rectal advancement flap for the treatment of complex cryptoglandular anal fistulas: a systematic review and metaanalysis. Int J Colorectal Dis 2017;32:599-609. 